\title{
Experimental analysis of mechanical response of stabilized occipitocervical junction by 3D mark tracking technique
}

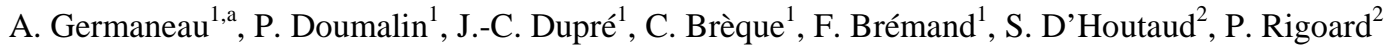 \\ ${ }^{1}$ Institut Pprime UPR 3346, CNRS - Université de Poitiers - ENSMA \\ S.P.2M.I., Boulevard Marie et Pierre Curie, Téléport 2, 86960 Futuroscope Chasseneuil, France \\ ${ }^{2}$ CHU La Milétrie Poitiers \\ 86000 Poitiers, France
}

\begin{abstract}
This study is about a biomechanical comparison of some stabilization solutions for the occipitocervical junction. Four kinds of occipito-cervical fixations are analysed in this work: lateral plates fixed by two kinds of screws, lateral plates fixed by hooks and median plate. To study mechanical rigidity of each one, tests have been performed on human skulls by applying loadings and by studying mechanical response of fixations and bone. For this experimental analysis, a specific setup has been developed to impose a load corresponding to the flexion-extension physiological movements. 3D mark tracking technique is employed to measure 3D displacement fields on the bone and on the fixations. Observations of displacement evolution on the bone according to the fixation show different rigidities given by each solution.
\end{abstract}

\section{Introduction}

Craniocervical junction can be rendered unstable because of various disease problems, stabilization from occipito-cervical fixation is necessary. Stabilization of the craniocervical junction consists in a rigid fixation between the occiput and upper cervical spine by using plates, rods and screws. Several works have already validated the contribution of the craniocervical junction according different pathologies [1-6].

Different kinds of fixations have been developed [5-8] in order to insure the stability of the junction like rods fixed on lateral plates associated screws or with hooks or on a median plate with screws. The present study is about the fixation around the occiput bone because of the dilemma imposed to surgeons: safe surgery and stability. Indeed, fixations involve more or less safely operation according to the location of screw implantations. The external occipital protuberance is the thickest part of the occiput and that occipital thickness decreases in a radial fashion laterally and inferiorly from this point. The variable local bone thickness of the occiput, combined with the location of the dural sinuses, dictates the safe working length for occipital screws, thus potentially affecting fixation strength and rigidity.

\footnotetext{
a e-mail : arnaud.germaneau@univ-poitiers.fr
} 
The objective of this study is to analyse mechanical response of four different kinds of occipitocervical fixations: lateral plates fixed with screws, lateral plates fixed with screws with a smaller pitch, lateral plates fixed with hooks and median plate fixed with screws. For that, mechanical tests are performed on human cadaveric specimens equipped with these fixations and displacements of the occipital bone and of the fixations (plates, screws, hooks, rods) have to be measured. Imposed mechanical loading has to correspond to human physiological movements of flexion-extension. In the present case displacement fields have to be measured on 3D surfaces and so a 3D non-invasive technique, the 3D mark tracking technique, is employed. This paper shows the experimental analysis of occipito-cervical fixation from mechanical loads from $3 \mathrm{D}$ displacement measurements.

\section{Specimen preparation and fixation techniques}

Four human cadaveric skull specimens are studied in this paper, each one is equipped by a kind of occipito-cervical fixation. These specimens were obtained fresh frozen at $-20^{\circ} \mathrm{C}$ and then thawed overnight. After thawing, skulls were extracted and cleared of soft tissue. In each specimen, the calvaria was sawed and the brain and soft tissues were removed. Anterior skull structures, including the mandible, were removed too. Then, spine and the cervical vertebras were removed and are substituted by a metallic vertebra (Figure 1-a) on which load is applied. For each skull, superior sawed part was potted in rigid polyurethane resin.

Occipital fixation techniques studied in this paper are shown in the Figure 1. Occipitocervical fixations are constituted by a $3 \mathrm{~mm}$-diameter rod. The straight side of the rod is fixed to cervical spine and the other extremity is a plate which is fixed at the occipital bone of the skull. This latter junction can be made with screws directly implanted in the bone or hooks fixed in a hole made in the skull. The first skull is equipped with lateral plates fixed by one kind of screws (Figure 1-b), the second one with lateral plates fixed by another kind of screws (Figure 1-c), the third one with lateral plates fixed by hooks (Figure 1-d) and the last one with a median plate fixed by screws (Figure 1-e). Diameter of screws implanted in the skull is $4 \mathrm{~mm}$ and the length is adapted to the local thickness of the bone. For the second solution, to implant hooks, a hole with a diameter of $8 \mathrm{~mm}$ has to be drilled on both sides of the external occipital crest.

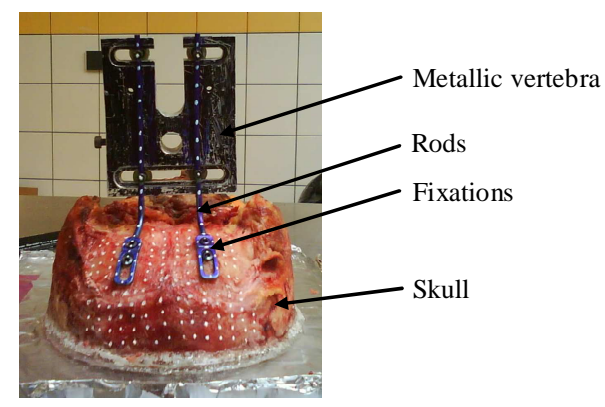

(a) Metallic vertebra and fixations

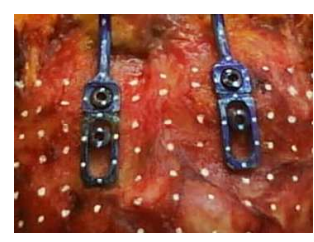

(b) Skull 1: lateral plates and screws 1

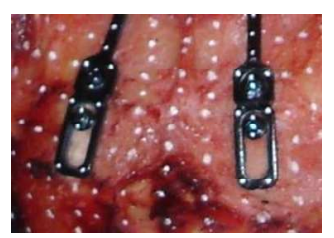

(c) Skull 2: lateral plates and screws 2

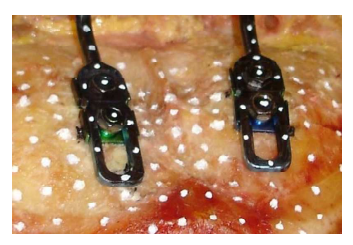

(d) Skull 3: lateral plates and hooks

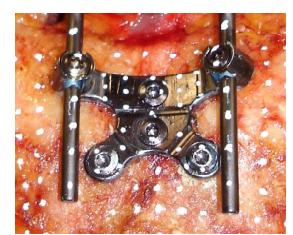

(e) Skull 4: median plate and screws

Fig. 1. Studied specimens equipped with different kinds of fixations 


\section{Mechanical testing}

To study mechanical loading of the occipital junctions, loading has to be imposed on rods where cervical vertebras are normally fixed. For that, a metallic vertebra has been built to substitute the real ones (Figure 1-a). Then, the load is applied directly on the metallic vertebra with a classical tensile test setup (Figure 2-a). To study mechanical response of occipital fixations, it is necessary to impose a mechanical loading corresponding to the flexion-extension physiological movements of human head. These movements can be reproduced by imposing loads corresponding to moments. As a tensile test setup is employed, moments are created by imposing a force with a level arm which is the distance connecting the load axis to the point of force application (Figure 2-b). To control the value of imposed moment, this distance is measured in each case during the mechanical test. The direction of the force has to be maintained according the same axis $(y)$ during the test in order to always create a moment according to the same axis $(x)$ and so $2 \mathrm{D}$ translation stages are placed in a plane orthogonal to the direction of the imposed force (Figure 2-a)).

Each specimen is cyclically loaded ramping from -6 N.m to +6N.m. To impose these values, displacement is in real-time controlled and imposed with a speed of $15 \mathrm{~mm} / \mathrm{min}$ and displacement is measured by 3D mark tracking technique with a frequency of $0.5 \mathrm{~Hz}$.

(a)

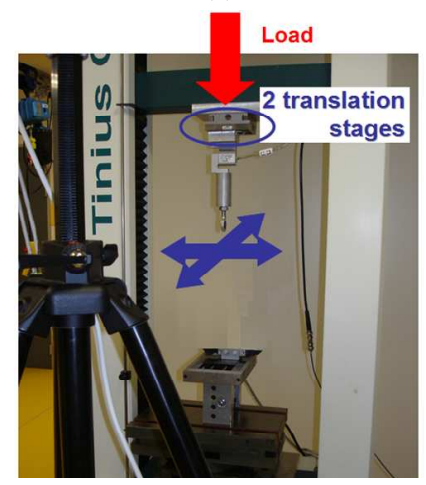

(b)

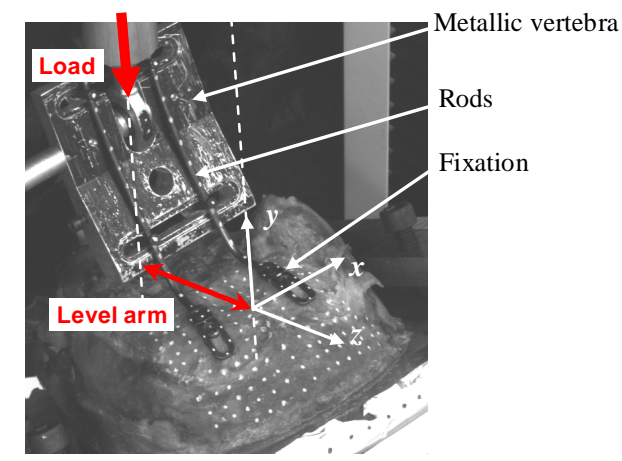

Fig. 2. Loading setup

\section{3D mark tracking method}

Displacement has to be measured during the mechanical loading on the full surface of the posterior skull but also on the fixations and on the rods. Furthermore displacement of the metallic vertebra has also to be measure to know the imposed moment value. So a field measurement method has to be employed and according to the morphology of the specimens and also the geometry of the setup, this method has to be able to measure 3D displacements without contact. For that, only an optical method can be used and as the displacement has to be measured in different points of the setup (skull, fixations, rods, resin), 3D mark tracking technique has been chosen. This method enables to measure 3D displacements of marks (natural or artificial) deposed on surfaces. The observation is based on a stereoscopic vision to obtain evolution of mark positions in the space. Two CCD cameras (noted 1 and 2) are employed (Figure 3). To determine 3D position of each mark, the first step consists in computing the position of each one $\left(\underline{X}_{i}, i=1,2\right)$ in each bench mark associated to each CCD camera. In fact, displacements of marks are tracked simultaneously in the bench mark of each camera.

Process to compute mark positions according to each CCD view is the same than the one developed in the 2D case [9]. In recorded images, intensity of marks has to be contrasted compared 
to the intensity $I(x, y)$ of the studied surface. Marks are generally white on dark surfaces or black on bright-field and they can be isolated from the field by defining a threshold intensity value $\left(I_{t}\right)$ in a research zone around each point. This research zone is defined by its dimensions $\left(N_{x}, N_{y}\right)$ from the initial state. Then, the coordinates $\underline{X}_{k i}\left(x_{k i}, y_{k i}, z_{k i}\right)$ of one mark according to a coordinate system associated with one of both CCD cameras are given by equations (1):

$$
\begin{gathered}
x_{i}^{k}=\frac{\sum_{x=0}^{N_{x}} \sum_{y=0}^{N_{y}} x_{i} \cdot\left(I\left(x_{i}, y_{i}\right)-I_{t}\right)}{\sum_{x=0}^{N_{x}} \sum_{y=0}^{N_{y}}\left(I\left(x_{i}, y_{i}\right)-I_{t}\right)} \\
y_{i}^{k}=\frac{\sum_{x=0}^{N_{x}} \sum_{y=0}^{N_{y}} y_{i} \cdot\left(I\left(x_{i}, y_{i}\right)-I_{t}\right)}{\sum_{x=0}^{N_{x}} \sum_{y=0}^{N_{y}}\left(I\left(x_{i}, y_{i}\right)-I_{t}\right)}
\end{gathered}
$$

Where $i$ gives the considered CCD camera, $I\left(x_{i}, y_{i}\right)$ is the light intensity of the pixel of coordinates $\left(x_{i}, y_{i}\right)$ and $I_{t}$ is the threshold intensity.

Then, knowing positions of marks in each CCD coordinate system, the second step consists in computing coordinates of marks in space $\left(\underline{X}_{R}\right)$ through a transition matrix $\boldsymbol{R}_{i}$ :

$$
R_{i} \underline{X}_{R}=\underline{X}_{i} \quad(i=1,2)
$$

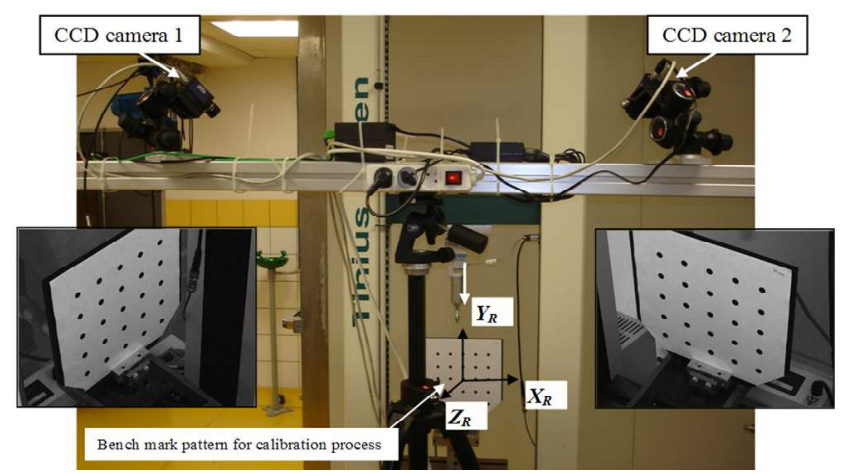

Fig. 3. 3D mark tracking setup.

To determine transition matrix from each local CCD bench mark to general space bench mark, a calibration process with a grid is necessary (Figures 3 and 4). This pattern is a plate where there are spots with known positions and regularly spaced. The calibration process consists firstly in linking the same mark between both images recorded by each CCD camera. In a second time, knowing the geometry of the observed area and also the coordinate $\underline{X}_{i}$ in each CCD coordinate system (Figure 4), the transition matrix $\boldsymbol{R}_{i}$ can be computed. Once this process performed, the bench mark pattern can be removed and substituted by the studied sample and measurements can begin on cadaveric samples. Data measured are displacements fields on the skull but also on rods and on fixations (screws or hooks) between several loaded states. In this present study, displacement measurement uncertainty has been estimated around $0.01 \mathrm{~mm}$ from imposed displacement tests.

In the present case, 3D mark tracking technique has to be applied on bone skulls, tissues but also on rods, fixations and metallic vertebra. To obtain a sufficient contrast of spots according to the measured surfaces, a specific white-ink pen is employed to depose marks on the skull (Figure 1). Surfaces of metallic parts and fixations are dark painting and white marks are also deposed. 


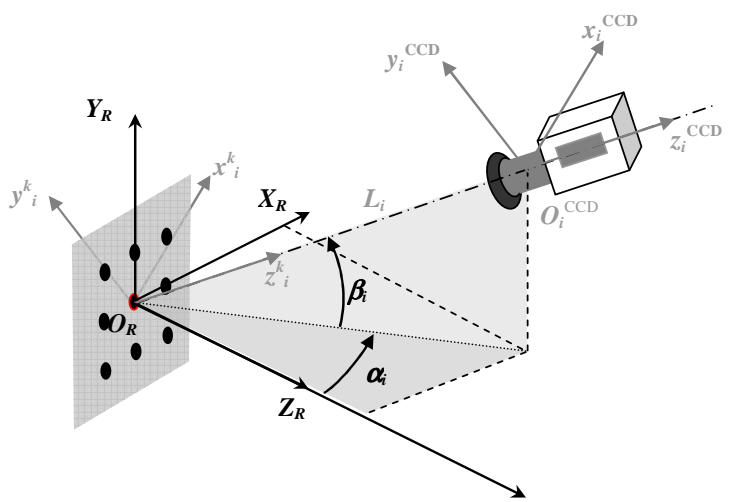

Fig. 4. Coordinate system for one of both CCD cameras

\section{Results}

3D displacement fields are measured on the full surface of the occipital bone according to the loading. Figure 5 shows components of displacement field measured on the skull equipped with hooks for a loading corresponding to an imposed moment equal to 5N.m. Globally, displacement is more significant according to the direction $y$ (which is the loading axis) than along the other directions. Displacement fields present some irregularities maybe because of several marks placed on tissues which remain on the bone surface and do not follow really bone movements. However, displacement values are low on the bone surface and seem to be larger near the fixations. Furthermore, global movement can be observed as seen on direction $x$. So, to study mechanical rigidity of the fixations, it is necessary to compare locally displacement values between fixations and bone and also between each kind of fixations.
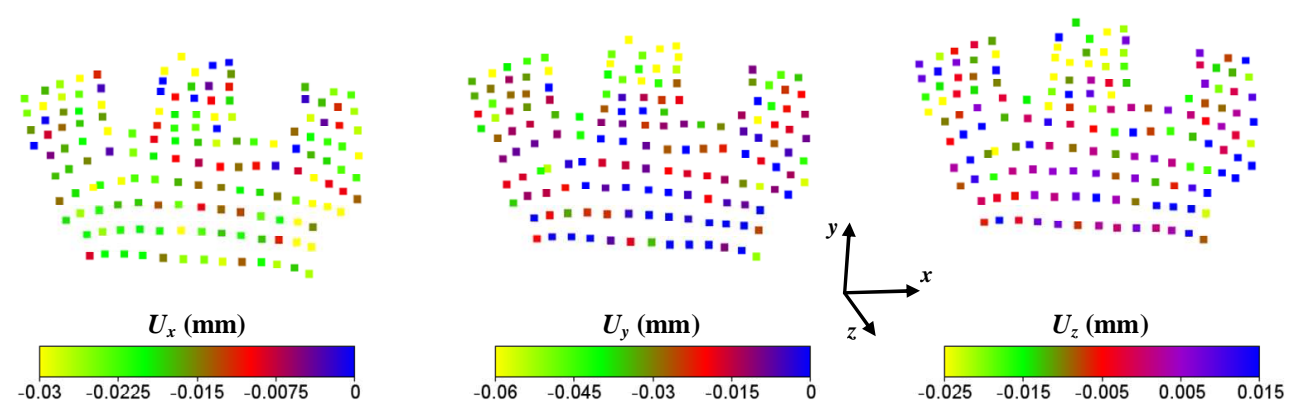

Fig. 5. Displacement field measured on the skull 3 equipped with hooks

To analyse more finely mechanical response of the fixations; three measurement points $(\mathrm{A}, \mathrm{B}, \mathrm{C})$ are analysed (Figure 6): one on the bone near the fixation (point A), another on the fixation plate (B) and the last on one screw (C). For skulls 1, 2 and 4, screws are directly fixed in the bone whereas for the skull 3 equipped with hooks, the hoock is fixed to the plate by a screw. Thus, these observations have to show displacement differences in the occipitocervical fixation which has to transmit mechanical loadings. 


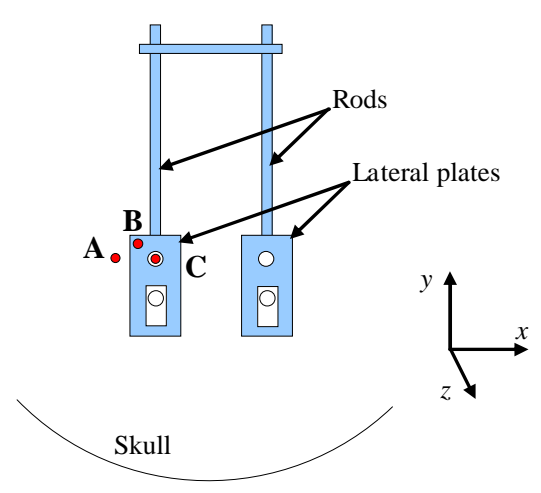

(a)

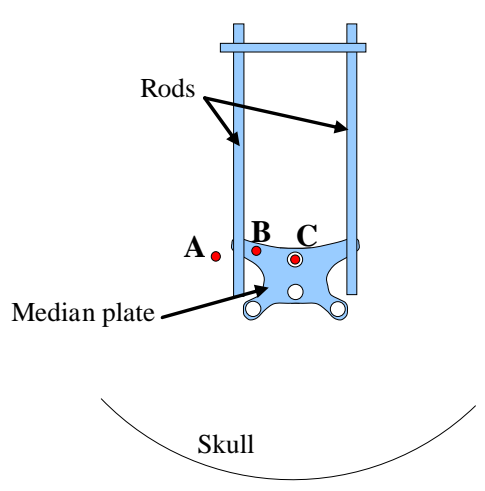

(b)

Fig. 6. Displacement analysis on three measurement points

Figure 7 shows evolution of displacement components on the three measurement points according to imposed moment values for the four skulls. For the skull 1, equipped with lateral plates fixed with screws, similar displacements are measured on the bone, on the plate and on the screw. The higher measured displacement, around $0.4 \mathrm{~mm}$, is along the direction $z$ which is a direction perpendicular to the occipital bone surface. In this first case, movements of the occipital bone are the same than the one of the plate and the screw. The occipital bone is deformed by the loading applied on the rods because of the very low thickness of the bone where the screws are implanted and the fixation insures a good rigidity.

Concerning the skull 2, also equipped with lateral plates but fixed with another kind of screws, displacements on the bone are very low. Measurements on the plates and on the screw show that there are only small movements with limited amplitudes of about $0.2 \mathrm{~mm}$. For this second skull, lateral plates and screws are implanted in the same area of the occipital bone than with the first skull that is to say where the bone thickness is very low. Nevertheless, the rigidity of the fixation is higher in the latter case because of the screw pitch which involves a better and a larger contact surface with the bone.

Bone displacements observed on the skull 3 equipped with hooks remain also low but significant displacements are measured on the fixations along directions $y$ and $z$ with values around $1.5 \mathrm{~mm}$ for the maximal loading. Significant displacements are logically observed on the screw which is not fixed in the bone but between the rod and the hook with this kind of fixation. Displacement values measured on the plate are of the same order than the ones observed the both fixations except according to the $y$-direction and only with positive values of the imposed moments. With this kind of fixation, hook is fixed with the bone by picking it along its thickness. If the hook is slightly larger than the bone thickness, there can remain a small gap with the load direction inversion, but once the gap is spent, load transmission is insured and the fixation appear rigid.

For the skull 4 equipped with a median plate, displacement values are also low for the points on the bone and on the screw. With this kind of fixation, screw is fixed in the external occipital crest which is the largest thickness part of the occipital bone. However, significant movements are observed on the plate and this is certainly due to the deformation of the plate involved by the loading. Indeed, plate is fixed with the bone only in its median part and rods are fixed in its lateral parts. The loading is performed on the rods, the median part of the plate is fixed in the bone and is very rigid, and so only the lateral parts of the plates are deformed. 

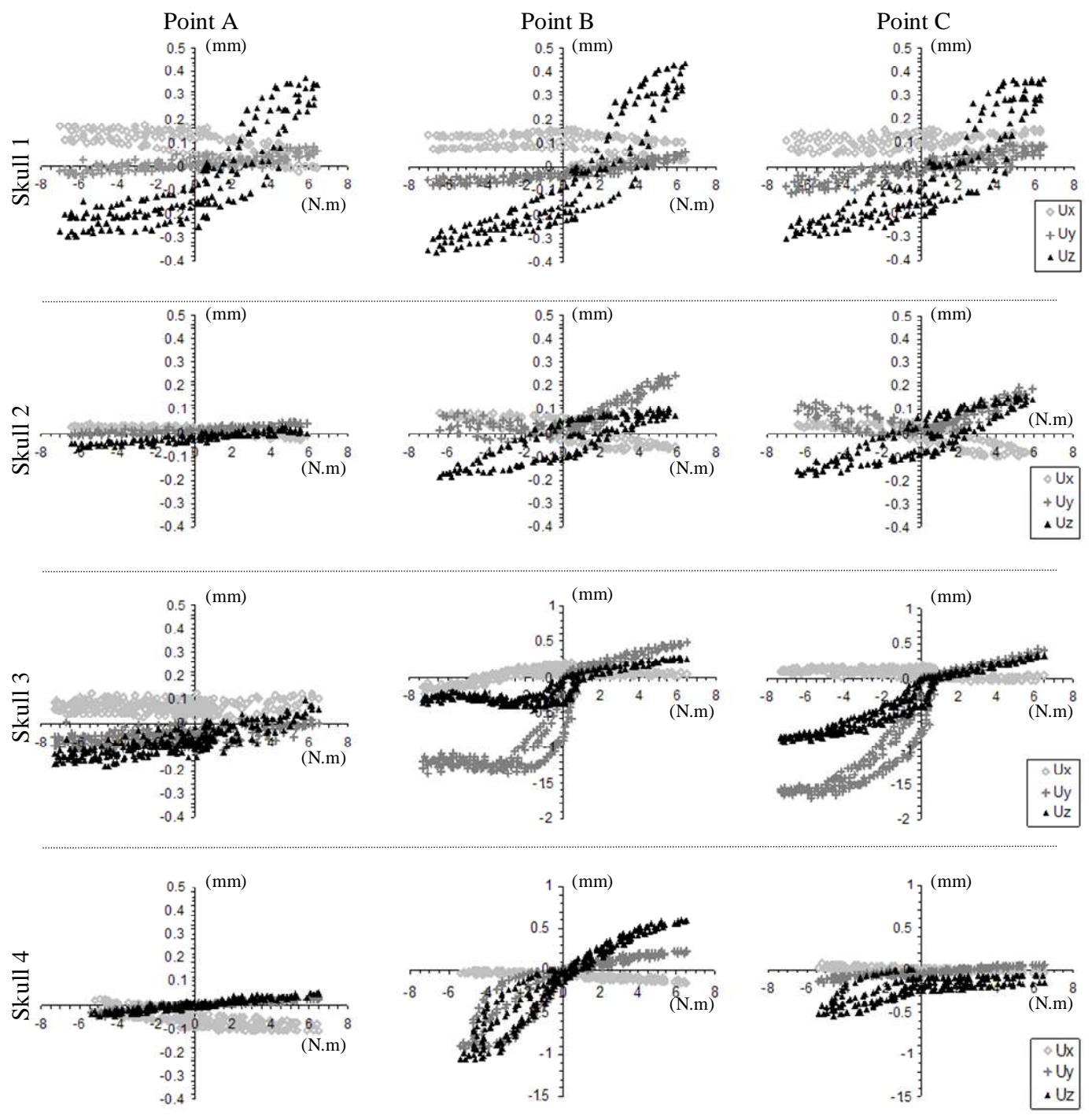

Fig. 7. Displacement analysis on three points

\section{Conclusion}

This paper shows experimental analysis of four kinds of occipitocervical junctions by a 3D optical measurement method. Indeed, 3D mark tracking technique has been adapted for this work and enables to measure 3D displacement fields on skulls equipped with fixations and solicited by a load corresponding to flexion-extension physiological movements. This study shows mechanical responses of these different stabilization techniques and enables to highlight rigidity of fixations according to the occipital bone.

Among these four kinds of fixations, different mechanical responses can be observed. The first kind of fixation presents a smaller rigidity than the three other ones maybe due to the screws fixed in the smallest thickness of the occipital bone. The skull equipped with the median plate shows another problem concerning the deformation of the fixation. Indeed, the fixation within the bone is highly 
rigid and with a cyclic loading the plate is deformed cyclically. Then, fixation by hook seems to transmit loading correctly but there can be a gap between the hook and the bone thickness involving a low rigidity which could cause a local bone spoiling. At last, the fusion which seems to show the best accordance of displacement field between the bone and the fixations is the one which employed lateral plates fixed with specific screws. These screws allow a rigid fixation in the bone in spite of its low thickness and insure the transmission of the loading without too large deformation of the bone or of the plates.

These observations show different mechanical rigidities of the occipital fixations. However, these first results have to be confirmed or invalidated by a statistical study made from other mechanical tests performed on several skull series. Furthermore, to determine completely mechanical behaviour of the junctions, it is necessary now to extend the study by applying loads corresponding to others physiological movements: lateral bending and axial rotation.

\section{References}

1. P. Korovessis, P. Katonis, A. Aligizakis, J. Christoforakis, A. Baikousis, Z. Papazisis, Eur. Spine J. 10, 5 (2001)

2. S. Lee, J. Chen, S. Lee, J. Clin. Neurosci. 13, 2 (2006)

3. P. Apostolides, C. Dickman, J. Golfinos, S. Papadopoulos, V. Sonntag, Spine 21, 14 (1996)

4. C. Huckell, J. Buchowski, W. Richardson, D. Williams, J. Kostuik, Clin. Orthop. Relat. Res. 359 (1999)

5. C. Wolfla, Spine J. 6, 6 Suppl. (2006)

6. J. Vender, A. Rekito, S. Harrison, D. McDonnell, Neur. Focus 16, 1 (2004)

7. A. Faure, E. Bord, R. Monteiro Da Silva, A. Diaz Saldaña, R. Robert, Eur. Spine J. 7, 1 (1998)

8. T. Mingsheng, W. Huimin, J. Xin, Y. Ping, W. Hongyu, Y. Feng, Eur. Spine J. 16, 12 (2007)

9. $\quad$ N. Bretagne, V. Valle, J.-C. Dupré, NDT\&E Int. 38 (2005) 